\title{
GPPS-CH-2020-0045
}

\section{CHALLENGES OF COMPRESSING HYDROGEN FOR PIPELINE TRANSPORTA- TION WITH CENTRIFUGAL-COMPRESSORS}

\author{
Sebastian Schuster \\ Chair of Turbomachinery \\ University of Duisburg-Essen \\ Duisburg, Germany \\ s.schuster@uni-due.de
}

\author{
Hans Josef Dohmen \\ Chair of Turbomachinery \\ University of Duisburg-Essen \\ Duisburg, Germany \\ hans-josef.dohmen@uni-due.de
}

\author{
Dieter Brillert \\ Chair of Turbomachinery \\ University of Duisburg-Essen \\ Duisburg, Germany \\ dieter.brillert@uni-due.de
}

\begin{abstract}
In this paper, the challenges of compressing hydrogen $\left(\mathrm{H}_{2}\right)$ for transportation mainly in pipelines utilizing centrifugalcompressors are outlined. The focus is on a continuously increasing $\mathrm{H}_{2}$ fraction in existing natural gas pipeline networks. Thereby, this paper raises consciousness for a new research area. Based on basic thermodynamic analysis, mean-line and computational fluid dynamics (CFD), the challenges and required measures to reach pressure ratios in a single-stage similar to compressors for heavier gas molecules are highlighted. Furthermore, all results are compared to $\mathrm{CH}_{4}$. Increasing the rotational speed or, at least partially, using counter rotating rotors emerges as possible solutions. Thereby, an economically implementation is possible and brings $\mathrm{H}_{2}$ one step closer to a storage option for energy converted from renewable sources. As a case study, a singlestage centrifugal-compressor for re-compression in pipelines is designed and analysed with $3 D C F D$. The operation of the compressor in conjunction with the pipeline is investigated. In conclusion, a pipeline equipped with centrifugal-compressors using variable speed control can deliver constant heating power for pure $\mathrm{CH}_{4}$ and pure $\mathrm{H}_{2}$. To achieve the required circumferential speeds, advanced materials are necessary and future research and development activities have to focus on the application of such materials to centrifugal-compressors.
\end{abstract}

\section{NOMENCLATURE}

$a$
Speed of sound

Velocity in stationary frame

Counter rotating rotor

Enthalpy

$\begin{array}{ll}\Delta h_{s} & \text { Isentropic enthalpy change } \\ \Delta h_{s t g} & \text { Actual enthalpy change in a stage } \\ M & \text { Molar mass } \\ p & \text { Pressure } \\ R_{m} & \text { Universal molar gas constant } \\ T & \text { Temperature } \\ u & \text { Circumferential velocity } \\ v & \text { spec. volume } \\ w & \text { Velocity in relative frame } \\ w_{t} & \text { Work } \\ \gamma & \text { Isentropic exponent } \\ \phi_{i} & \text { Volume fraction of component } i \\ \varphi & \text { Through-flow coefficient } \\ \psi_{h} & \text { Enthalpy coefficient }\end{array}$

\section{INTRODUCTION}

Hydrogen $\left(\mathrm{H}_{2}\right)$ is discussed as an option to store the energy converted from renewable sources as outlined by the German Federal Ministry for Economic Affairs and Energy [1]. This storage technology can complement other technologies and in certain cases can have advantageous such as: transport in existing pipelines, decoupling of storage capacity and power output (compared to most batteries). Furthermore, $\mathrm{H}_{2}$ can be used for direct electricity conversion, e.g., in fuel cells [2], conversion into mechanical energy [3], and industrial processes, e.g., in blast furnaces [4].

Utilizing $\mathrm{H}_{2}$ as storage medium requires generation, transportation, and conversion into electrical, mechanical or heat energy. This paper focuses on onshore transportation and more in

This work is licensed under Attribution 4.0 International (CC BY 4.0) See:

https://creativecommons.org/licenses/by/4.0/legalcode 
detail on pressurizing and re-pressurizing $\mathrm{H}_{2}$ for transportation in pipelines with centrifugal-compressors. In this paper, a case study for a pipeline with a nominal pressure of 40 bar and recompression after a pressure drop of 20 bar is conducted.

$\mathrm{H}_{2}$ is generated at pressure levels between $1-80$ bar [5]. The pipeline pressure is in a range between 30 bar to 100 bar [6] and hence an initial pressurisation after generation can be necessary. On the other hand, pressure losses in the pipeline have to be compensated by re-compression every few hundred kilometres. Results of previous studies on the operation of centrifugalcompressors in pipelines suggest an increase of the required number of stages for the compression of $\mathrm{H}_{2}$ in comparison to $\mathrm{CH}_{4}$ [7-9]. The reasons for this are outlined in the next paragraph. This paper continues the research on $\mathrm{H}_{2}$ transportation by:

(i) formulating the research questions with basic thermodynamic relations

(ii) designing a centrifugal-compressor stage for re-compression of gases in pipelines

(iii) comparing $\mathrm{H}_{2}$ to $\mathrm{CH}_{4}$ transportation in pipelines by means of 3D CFD.

Finally, future research aims are formulated based on these investigations.

\section{Need for research and development from a thermody- namic perspective}

The differences and challenges introduced by switching from $\mathrm{CH}_{4}$ to $\mathrm{H}_{2}$ can readily be seen by considering the law of ideal gases. Whereby this approximation is justified by the compressibility factor $Z$ being close to unity. For instants, at typical pipeline conditions of 40 bar and $20^{\circ} \mathrm{C}$ the compressibility factors are $Z_{\mathrm{CH}_{4}}=0.93$ and $Z_{\mathrm{H}_{2}}=1.02$, respectively. More detailed diagrams of $Z$ and isobaric heat capacity $c_{p}$ are given in Appendix A.

The isentropic exponent $\gamma$ of $\mathrm{CH}_{4}$ is 1.3 and of $\mathrm{H}_{2}$ is 1.4. Thus, the ratio between isentropic outlet to inlet temperature is similar for the same pressure ratio and follows as:

$$
\frac{T_{2}}{T_{1}}=\left(\frac{p_{2}}{p_{1}}\right)^{\frac{\gamma-1}{\gamma}}
$$

However, the molecular weight $M$ is quite different with 16 $\mathrm{kg} / \mathrm{kmol}$ for $\mathrm{CH}_{4}$ and $2 \mathrm{~kg} / \mathrm{kmol}$ for $\mathrm{H}_{2}$. In turn, the required isentropic enthalpy difference for $\mathrm{H}_{2}$ is roughly 8 times higher and follows as:

$$
\Delta h_{s}=\frac{\gamma}{\gamma-1} \frac{R_{m}}{M} T_{1}\left(\left(\frac{p_{2}}{p_{1}}\right)^{\frac{\gamma-1}{\gamma}}-1\right)
$$

In contrast, the achievable enthalpy change in an adiabatic centrifugal-compressor stage is mostly independent of the fluid and for a repeating stage design and radial oriented blades at the outlet follows as:

$$
\Delta h_{s t g}=w_{t}-\frac{c_{3}^{2}-c_{1}^{2}}{2}=u_{2} c_{u 2}=u_{2}^{2}
$$

Finally, the number of stages can be computed by dividing the result of eq. 2 by the result of eq. 3 .

From the above equations three main aspects regarding the enthalpy change can be deduced. The enthalpy change for a given pressure ratio (eq. 2) increases with:

(i) decreasing molar weight $M$,

(ii) increasing inlet temperature $T_{1}$,

while the enthalpy change in the compressor (eq. 3) increases with:

(iii) increasing rotational speed $\omega=u_{2} / r_{2}$.

These results - although not surprising - are the backbone for answering the questions:

(i) How many stages are required if only the gas changes?

(ii) What measures can be taken to reduce the number of stages?

(iii) Which research questions and development steps arise from these measures?

\section{PIPELINE REQUIREMENTS AND COMPRESSOR CON- STRAINTS}

The ultimate goal of a gas pipeline network is to deliver a defined amount of energy to the end users. In such a pipeline, the task of the centrifugal-compressor is to compensate the pressure loss caused by friction in the pipeline for a given energy flow rate.

\section{Number of compressor stages and heating value}

To compute the required number of compressor stages, at first a case is considered where the rotational speed and the outlet flow angle are kept constant. The nominal pipeline pressure is 40 bar and pressure losses of 20 bar need to be compensated at a constant mass flow rate.

The resulting number of compressor stages computed with eqs. 2 and 3 are depicted in Fig. 1 together with the mixture caloric values as a function of the volumetric fraction of $\mathrm{H}_{2} \phi_{\mathrm{H}_{2}}$ in the pipeline. The fluid is treated as a mixture of ideal gases and the spec. (higher) heating value of $\mathrm{CH}_{4}$ is $55.5 \mathrm{MJ} / \mathrm{kg}$.

Seven times the number of stages are required for transportation of pure $\mathrm{H}_{2}$ as can be seen from Fig. 1 (black dashed-dotted line). The specific heating value (red bold line) increases while the volumetric heating value (red dashed line) decreases. In general, volumetric values have a stronger impact on the pipeline and on the compressor since pressure loss and compressor operating point are related to the volume flow rate due to the velocity. Therefore, volumetric values are proposed as the quantity to compare results between $\mathrm{CH}_{4}$ and $\mathrm{H}_{2}$. Whereas, for transportation in vessels, trucks or ships spec. values might be more relevant.

To ensure constant heating power, either the volume flow rate or the pressure need to be increased. Both options need to be considered in conjunction with the compressor and the pipeline. In the following, this paper focuses on increased volume flow rates. Further, measures to increase the single-stage work input need to be tested as well. According to eq. 3, work input increases with circumferential velocities $u_{2}$ and hence with rotational speed or with flow turning, e.g., $c_{u 2}$. Both options are investigated in detail in the following paragraphs. 


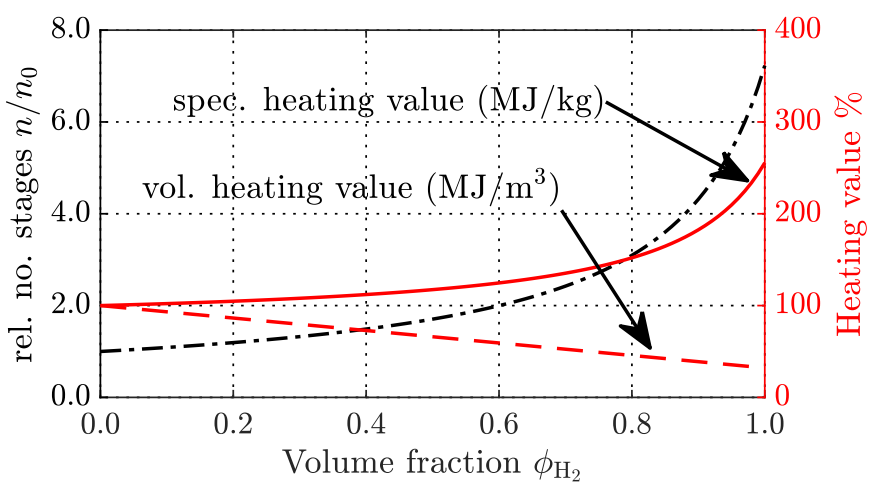

FIGURE 1: Number of compressor stages and heating value for a mixture of $\mathrm{H}_{2}$ and $\mathrm{CH}_{4}$ with constant mixture mass flow rate.

\section{Case 1 - Increasing rotational speed}

The impact of increased rotational speed on the energy conversion can be explained with the velocity triangles in Fig. 2. In order to achieve higher enthalpy changes without altering the blade angles, the velocity triangles need to be scaled up. Thereby, the meridional velocity $c_{m}$ increases and so does the volume flow rate. This can be beneficial in order to have a constant heating power delivered by the pipeline.

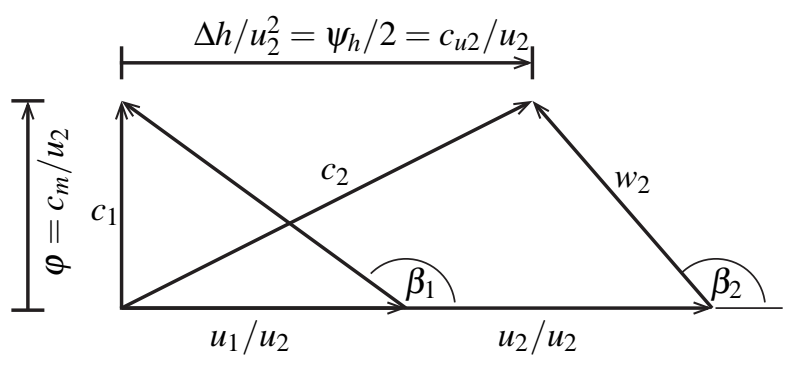

FIGURE 2: Velocity triangles of a centrifugal-compressor with repeating stage design at impeller inlet (1) and impeller outlet (2).

From an aerodynamic point of view, the circumferential velocity is only limited by the speed of sound $a$ of a fluid. Supersonic conditions are normally avoided $\left(c_{2}<a\right)$ in centrifugalcompressors resulting in $u_{2} \lesssim 0.8 a$. The speed of sound in turn is a function of the isentropic exponent and the molecular weight and calculated as:

$$
a=\sqrt{\gamma \frac{R_{m}}{M} T}
$$

This can be inserted with the restriction $u_{2}=0.8 a$ into eq. 3 and the number of required stage $n$ follows as:

$$
n=\frac{\frac{\gamma}{\gamma-1}\left[\left(\frac{p_{2}}{p_{1}}\right)^{\frac{\gamma-1}{\gamma}}-1\right]}{0.64 \gamma}
$$

From this eq. the conclusion emerges that for constant circumferential Mach-number $M a_{u}=u_{2} / a$, the required number of stages is only a function of the pressure ratio and a weak function of the isentropic exponent of the mixture $\gamma$. In Fig. 3, the required number of stages for constant pressure ratio (black dashed line) and resulting speed of sound variation (red bold line) are shown. The increasing speed of sound overcompensates the required enthalpy due to the variation of $\gamma$. Certainly, the mechanical stresses increase dramatically with circumferential speed since the centrifugal force is proportional to the square of the circumferential velocity. In conclusion, the higher speed of sound of $\mathrm{H}_{2}$ allows to increase the rotational speed. Thereby, the number of compressor stages can be reduced. On the other hand, increasing the rotational speed requires a detailed analysis of the mechanical stresses.

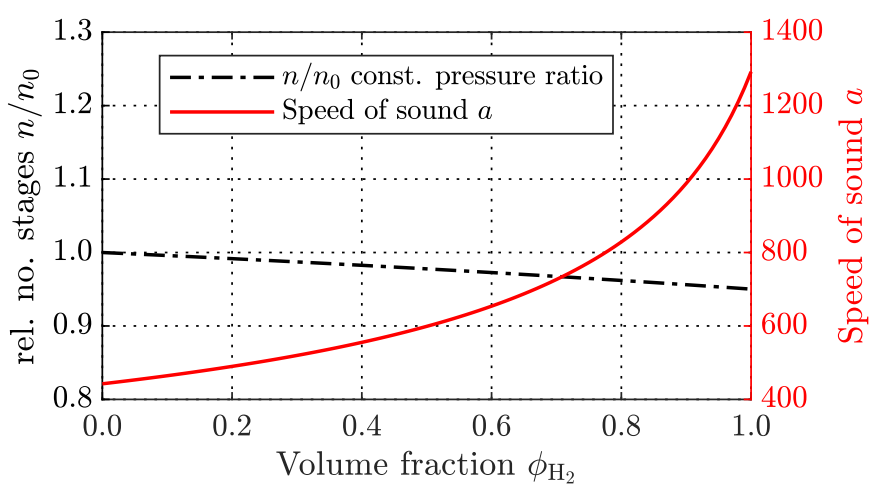

FIGURE 3: Required number of compressor stages for constant circumferential Mach-number.

\section{Case 2 - Increasing flow turning}

The work input can also be increased by a higher flow turning as can be seen from the Euler work equation. In this context forward curved blades can be used since for this type of blade $c_{u 2}$ increases. On the other hand, higher losses are to be expected caused by the increased velocity. Alternatively, a second rotor placed directly after the first one and rotating in opposite direction can be applied as has been investigated for centrifugal-pumps by Tosin [10]. The resulting velocity triangles are depicted in Fig. 4.

The enthalpy coefficient of the entire compressor (consisting of both rotors) should be related to the second rotor outlet circumferential speed $u_{4}$ to account for the mechanical stresses. For a repeating stage design it follows as: 


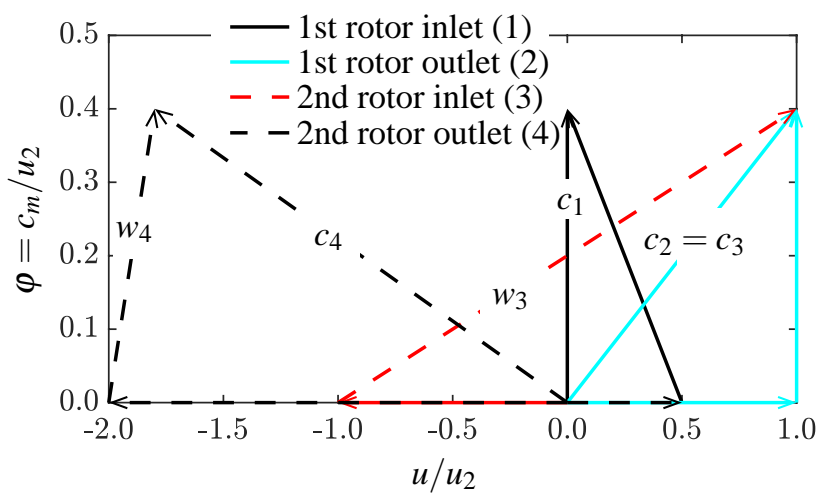

FIGURE 4: Velocity triangles of a counter rotating stage design (not to scale). Velocity vector definition $c=u+w$

$$
\psi_{h, c r}=\frac{u_{4} c_{u 4}-u_{3} c_{u 3}+u_{2} c_{u 2}-u_{1} c_{u 1}}{u_{4}^{2} / 2}
$$

For radially oriented blades at the outlet of the first and the second rotor as well as the assumption that the circumferential speed of the second rotor is twice the one of the first but with opposite sign, a maximum value of $\psi_{h, c r}=3$ can be achieved, where 2 is the limit for a conventional design. In comparison to the conventional stage, the rotational speed can be reduced by a maximum of $20 \%$. This follows directly from the definition of the enthalpy coefficient as:

$$
\frac{u_{4, c r}}{u_{2, c}}=\sqrt{\frac{\psi_{h, c}}{\psi_{h, c r}}}=0.8
$$

A disadvantage is the high de-acceleration ratio in the second rotor of $w_{4} / w_{3}=0.2$. Therefore, the compressor is highly loaded presumable resulting in an efficiency penalty. Reducing the blade loading results in smaller enthalpy coefficients as can be seen from Fig. 5 where a ratio of $w_{4} / w_{3}=0.2$ corresponds to radial oriented blades at the outlet of the second rotor. At least a ratio $w_{4} / w_{3}=0.5$ or lower is necessary to achieve a higher enthalpy change per stage at similar mechanical stresses. If high blade loadings are to be avoided, this type of compressor does not have a benefit. In conclusion, increasing the rotational speed seems to be more promising and is further analysed.

\section{CASE STUDY - PERFORMANCE MAP OF A CENTRIFUGAL-COMPRESSOR}

In this case study, a pipeline compressor is considered that re-compresses the gas to overcome the pressure losses of about 20 bar in a natural gas pipeline. The design parameters of the pipeline are: diameter of $1000 \mathrm{~mm}$, mean velocity of $7.5 \mathrm{~m} / \mathrm{s}$ for $\mathrm{CH}_{4}$ and pressure of 40 bar. A single-stage centrifugalcompressor is able to provide the required pressure ratio at least for $\mathrm{CH}_{4}$. The preliminary design tool presented by Hussein et al. [11] is used to design the impeller and the vaneless diffuser

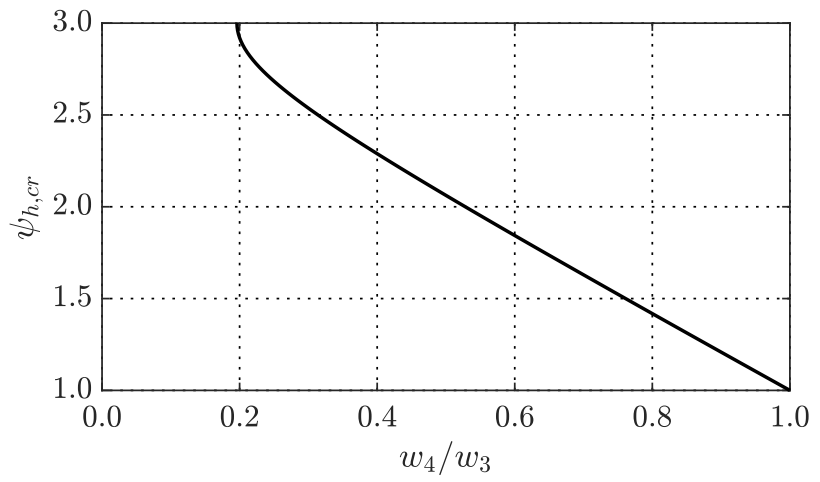

FIGURE 5: Achievable enthalpy coefficient as a function of the de-acceleration ratio.

of the single-stage centrifugal-compressor. For the investigation of the interaction with the pipeline, an inlet nozzle and a volute with exit diffuser are added. Thereby, the diameter at both ends is $1000 \mathrm{~mm}$, thus, providing a direct interface to the pipeline. The flow field is computed with Ansys CFX and more details are given in Tab. 1.

TABLE 1: Design parameters of the compressor and parameters for the computation

\begin{tabular}{l|c} 
Parameter & Value \\
\hline Tip diameter $d_{2} / \mathrm{mm}$ & 915 \\
Rotational speed / RPM & 8859 \\
Designed for & $\mathrm{CH}_{4}$ \\
Mass flow rate $/ \mathrm{kg} / \mathrm{s}$ & 200 \\
$p_{t 0} /$ bar & 20 \\
$T_{t 0} /{ }^{\circ} \mathrm{C}$ & 20 \\
Pressure ratio & 2 \\
$\beta_{2}$ & $90^{\circ}$ \\
Impeller blades & 11 \\
CFD solver & $\mathrm{CFX}$ \\
Turbulence & $\mathrm{SST}$ \\
Gas properties & Mixture of ideal gases \\
Temporal resolution & steady state
\end{tabular}

Performance maps are best depicted as energy conversion versus volume flow rate to compare a compressor working with different fluids. In non-dimensional form these are expressed as the enthalpy coefficient:

$$
\psi_{h}=\frac{\Delta h}{u_{2}^{2} / 2}
$$

the pressure coefficient:

$$
\psi_{y}=\frac{\int v d p}{u_{2}^{2} / 2}
$$


and the through-flow coefficient:

$$
\varphi=\frac{\dot{V} / A_{\perp}}{u_{2}}=\frac{c_{m}}{u_{2}}
$$

The resulting performance map based on changes of static values between inlet and outlet is depicted in Fig. 6. When the fluid is changed from $\mathrm{CH}_{4}$ (black bold lines) to $\mathrm{H}_{2}$ but rotational speed is constant (blue dotted lines) the slope of the curves change and $\psi_{y \text {,max }}$ is reduced. By increasing the rotational speed from $8859 \mathrm{rpm}$ to $25983 \mathrm{rpm}$, Mach-number similarity can be achieved (red dashed lines) and the slope and absolute values of the curves are much closer for both fluids. The remaining difference can be traced back to the different Reynolds-numbers computed with the impeller chord length and being $\operatorname{Re}_{\mathrm{CH}_{4}}=4.5 \mathrm{Mio}$. and $R e_{\mathrm{H}_{2}}=2.1$ Mio. for $\varphi=0.2$.

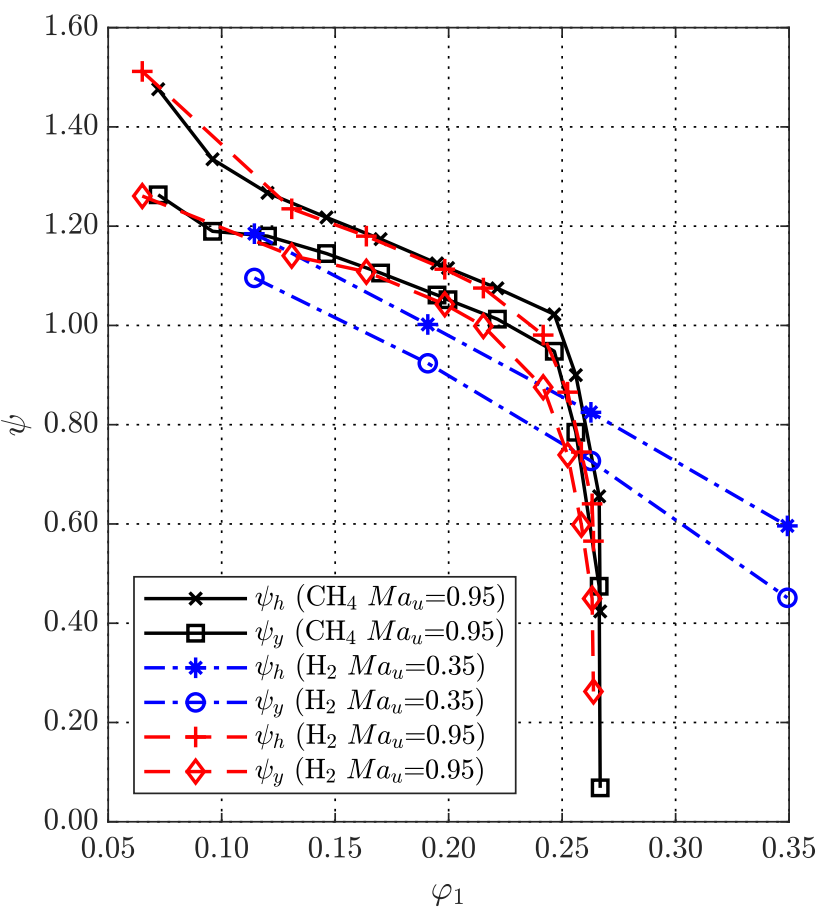

FIGURE 6: Performance map for a single-stage centrifugalcompressor based on static values for enthalpy and pressure.

Fig. 6 leads to the conclusion that the Reynolds-number effect is relatively small but the Mach-number effect is much stronger as can be observed in the same figure. Furthermore, Mach-number similarity also results in a similar pressure ratio, which follows from eqs. 2 and 4 and from the similar polytropic efficiency for the adiabatic process calculated as:

$$
\eta_{p}=\frac{\psi_{y}}{\psi_{h}}
$$

These results clearly show that similar pressure ratios for $\mathrm{H}_{2}$ compressors can be achieved if the circumferential speed can be increased up to $1300 \mathrm{~m} / \mathrm{s}$. Furthermore, the volume flow increases (what can be seen from the increasing through-flow coefficient $\varphi$ ). In turn, higher volume flow rates increase the power delivered by the pipeline but also might increase the pressure losses in the pipeline. In the next paragraph the interaction between the pipeline (in terms of pressure losses) and the compressor is investigated.

\section{Interaction between compressor and pipeline}

The pipeline of this case study is modelled as a straight tube so that pressure losses can be calculated from the pipe friction factor $\lambda$ obtained from Reynolds-number correlations, e.g., Schlichting [12]. Pressure losses are computed with pipeline inlet conditions neglecting the change of density and velocity in the pipeline. For $\mathrm{CH}_{4}$ the pressure drops from the initial pressure of 40 bar to 20 bar, which corresponds to a pipeline length of about $300 \mathrm{~km}$. To keep a constant heating power with changing mixture composition, the mean velocity (equals the pipeline inlet velocity) is increased and the impact on the resulting pressure loss is depicted in Fig. 7. Initially the pressure loss increases but afterwards decreases for volume fractions larger than 0.85 . The slope of the curve is the result of two effects acting in opposite direction: mean velocity increases but density decreases. Also the Reynolds-number increases and $\lambda$ decreases with values between 0.0088 and 0.0096. A more detailed verification is given in Appendix B. In conclusion, the pressure losses are similar for all mixture compositions.

Pressure drop vs. velocity represents the system characteristics for which a matching compressor performance map is required. The velocity at the compressor nozzle inlet $c_{m 0}$ corresponds to the pipeline outlet velocity. This can be computed for the isothermal pipeline from the pressure drop and the law of ideal gases. Further, the pressure drop is expressed as isentropic flow work $\left(\int v_{s} d p\right)$ in order to indicate the required energy conversion and to get an easy to read diagram. The resulting system characteristic of the pipeline is depicted in Fig. 8 (line with variable color).

Compressor performance lines of constant rotational speed are computed for different mixture compositions. The rotational speed is varied until it intersects with the system characteristics at the corresponding mixture composition. The required rotational speed can be estimated with the presented similarity rules (eqs. 9-10) confirmed in Fig. 6 for this case and can be iteratively adjusted in the CFD calculations.

For four mixture compositions the results are shown in Fig. 8. For pure $\mathrm{CH}_{4}$ and pure $\mathrm{H}_{2}$ a feasible operation point can be found (between choke and surge limit). But for a volume fraction of $\phi_{\mathrm{H}_{2}}=0.8$ the compressor is working in chocked conditions before the required volume flow is achieved with the delivered volume flow being $4 \%$ lower than required. This is due to the higher pressure losses in the pipeline. This difference is relatively small and can be possibly mitigated by increasing the size of the compressor so that it operates closer to the surge line for pure $\mathrm{CH}_{4}$. 


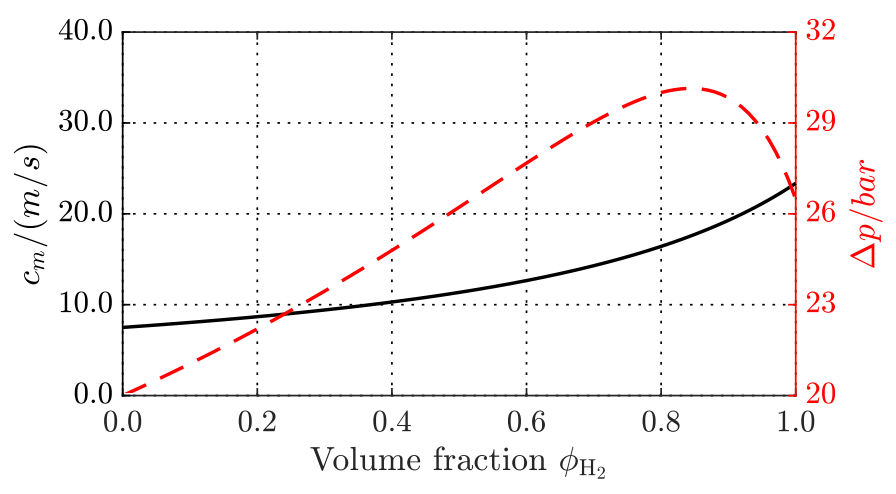

FIGURE 7: Mean gas velocity and pressure drop in a pipeline with constant heating power and variable mixture composition.

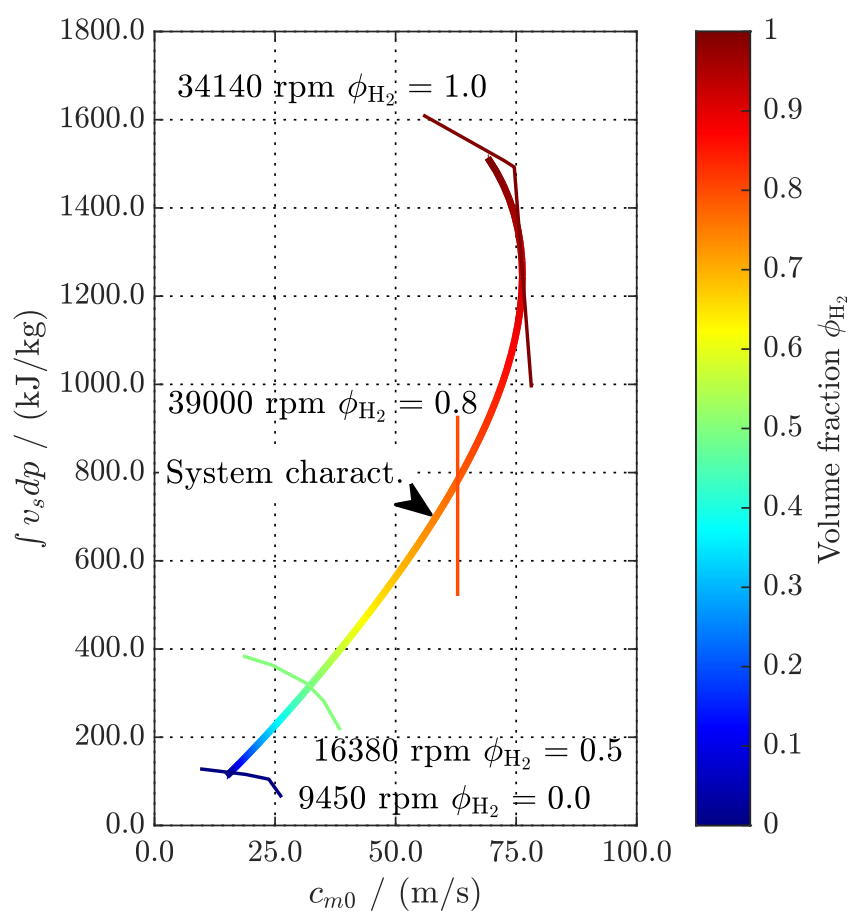

FIGURE 8: Performance map for a single-stage centrifugalcompressor and interaction with the system characteristics for constant heating power.

\section{Conclusions}

In this paper, the use of centrifugal-compressors for the transport of $\mathrm{H}_{2}$ in pipelines has been discussed by means of basic thermodynamic analysis and by 3D CFD calculations. The interaction between the pipeline and the centrifugal-compressor has been analysed. Focus has been on the transport of $\mathrm{CH}_{4}$ and $\mathrm{H}_{2}$ mixtures.

Thermodynamically, the main difference is the molecular weight of $\mathrm{H}_{2}$ being only one eighth of $\mathrm{CH}_{4}$. This corresponds to a proportional increase of the required enthalpy change for a given pressure ratio. At the same time, the speed of sound increases by the square root of eight. Hence, a compressor can rotate about 2.8 times faster and in the end can have a similar pressure ratio for $\mathrm{CH}_{4}$ and $\mathrm{H}_{2}$.

For the transport of gases in pipelines, a careful definition of properties and values being compared is necessary to end up with relevant conclusions. This value is the heating power delivered to the customer. To keep the heating power constant, the mean pipeline velocity needs to be increased by a factor of about three when switching from $\mathrm{CH}_{4}$ to $\mathrm{H}_{2}$. At the same time, the pressure losses in the pipeline change only marginal. This has a positive effect on the interaction between compressor and pipeline. A stable operation point can be found for pure $\mathrm{CH}_{4}$ and pure $\mathrm{H}_{2}$ when the compressor can be operated with variable rotational speed. Thereby, the circumferential speed increases up to values of 1300 $\mathrm{m} / \mathrm{s}$ and posses a mechanical challenge.

The required circumferential speed can most likely not be reached with conventional materials such as Steel, Aluminium or even Titanium for which the circumferential speed limit in the order of $600 \mathrm{~m} / \mathrm{s}$. New materials such a fibre-reinforced materials need to be selected and developed for the specific needs.

In terms of technical readiness levels, in this paper level 1 'Basic principles observed' has been fulfilled by observing the thermodynamic difference between $\mathrm{CH}_{4}$ and $\mathrm{H}_{2}$ compression. Further level 2 'Technology concept formulated' has been reached by showing that variable rotational speed is suitable to extend the operation range from compression of pure $\mathrm{CH}_{4}$ to pure $\mathrm{H}_{2}$. In the next steps suitable materials and alterations of the compressor design to complement these materials need to be selected and tested in the lab to reach level 3 'Experimental proof of concept' and level 4 'Technology validated in lab'.

\section{REFERENCES}

[1] BMWI, 2019. Wasserstoff und Energiewende. https://www.bmwi.de/Redaktion/DE/Downloads/JL/kurzpapier-wasserstoff.pdf. Accessed: 29.01.2020.

[2] Heinzel, A., 2009. Fuel cells and hydrogen technology. Springer Berlin Heidelberg, Berlin, Heidelberg, pp. 368373.

[3] ScienceDirect, $2020 . \quad$ Hydrogen engines. https://www.sciencedirect.com/topics/engineering/hydrogenengines. Accessed: 29.01.2020.

[4] thyssenkrupp Steel Europe, 2019. World first in Duisburg as NRW economics minister Pinkwart launches tests at thyssenkrupp into blast furnace use of hydrogen. https://www.thyssenkruppsteel.com/en/company/sustainability/climate-strategy/. Accessed: 29.01.2020.

[5] Voitic, G., Pichler, B., Basile, A., Iulianelli, A., Malli, K., Bock, S., and Hacker, V., 2018. "Hydrogen production". In Fuel Cells and Hydrogen. Elsevier, pp. 215-241.

[6] Krieg, D., 2012. Konzept und Kosten eines Pipelinesystems zur Versorgung des deutschen Straßenverkehrs mit Wasserstoff. Schriften des Forschungszentrums Jülich, Reihe Energie und Umwelt, Energy und Environment, Band 144.

[7] Tellier, C., 1981. "Application of axial flow compressors for long distance pipeline transmission of hydrogen". International Journal of Hydrogen Energy, 6(4), pp. 413-422.

[8] Heshmat, H., Hunsberger, A., Ren, Z., Jahanmir, S., and Walton, J., 2010. "On the design of a multi-megawatt oil- 
free centrifugal compressor for hydrogen gas transportation and delivery: Operation beyond supercritical speeds". In Proceedings of the ASME 2010 International Mechanical Engineering Congress and Exposition.

[9] Bella, F. A. D., and Fairman, K. D., 2012. "Development of a $240,000 \mathrm{~kg} /$ day hydrogen pipeline centrifugal compressor for the department of energy's hydrogen delivery and production program". In Proceedings of the ASME 2012 International Mechanical Engineering Congress and Exposition, American Society of Mechanical Engineers.

[10] Tosin, S., 2016. Counter-rotating mixed-flow pumps design and analysis. Dissertation, Technische Universität Braunschweig.

[11] Hussein, I., Schuster, S., and Brillert, D., 2020. “A design tool for supercritical $\mathrm{CO} 2$ radial compressors based on the two-zone model". In Accepted for publiction at the ASME Turbo Expo, American Society of Mechanical Engineers.

[12] Schlichting, H., and Gersten, K., 2017. Boundary-Layer Theory. Springer Berlin Heidelberg.

[13] Lemmon, E. W., Huber, M. L., and McLinden, M. O., 2007. NIST Standard Reference Database 23: Reference Fluid Thermodynamic and Transport Properties-REFPROP, Version 8.0, National Institute of Standards and Technology.

\section{Appendix A: Compressibility factor of $\mathrm{CH}_{4}$ and $\mathrm{H}_{2}$ at typical pipeline conditions}

In this paper the properties of $\mathrm{CH}_{4}$ and $\mathrm{H}_{2}$ are evaluated with the equation of ideal gases and constant spec. heat capacity. This approach is valid at typical pipeline conditions since the compressibility factor is close to one and shows only moderate gradients as can be seen from Fig. 9 and Fig. 10. Further, the specific heat is almost constant in the investigated temperature and pressure range as can be seen from Fig. 11 and Fig. 12. The values shown have been evaluated from the real gas equations of state implemented in the REFPROP [13] database.

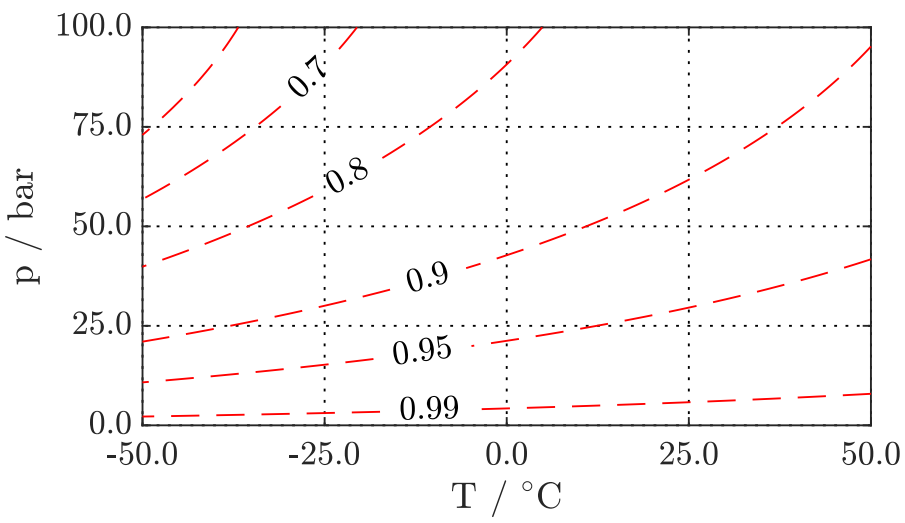

FIGURE 9: Compressibility factor of $\mathrm{CH}_{4}$

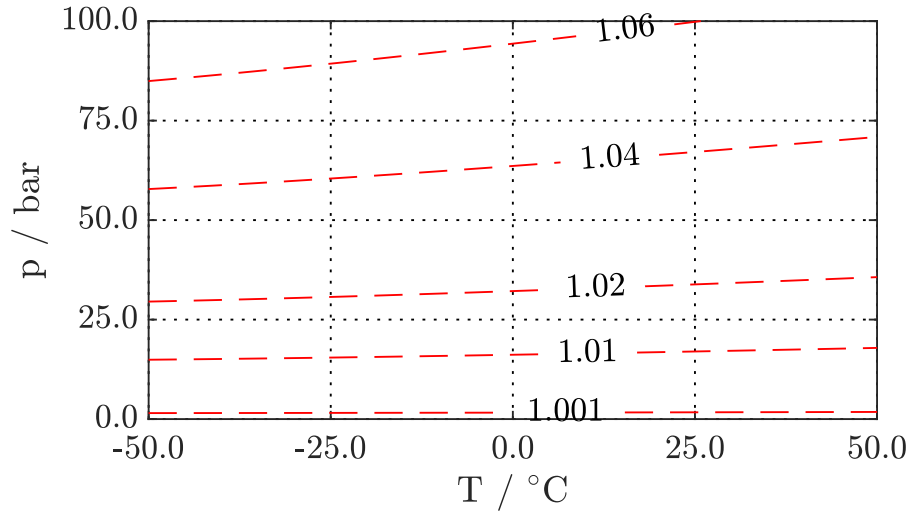

FIGURE 10: Compressibility factor of $\mathrm{H}_{2}$

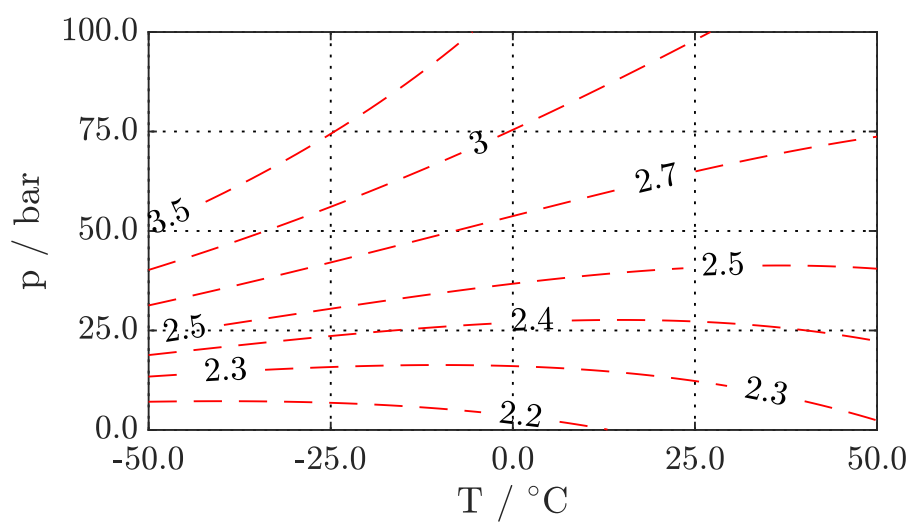

FIGURE 11: Spec. heat capacity of $\mathrm{CH}_{4}$

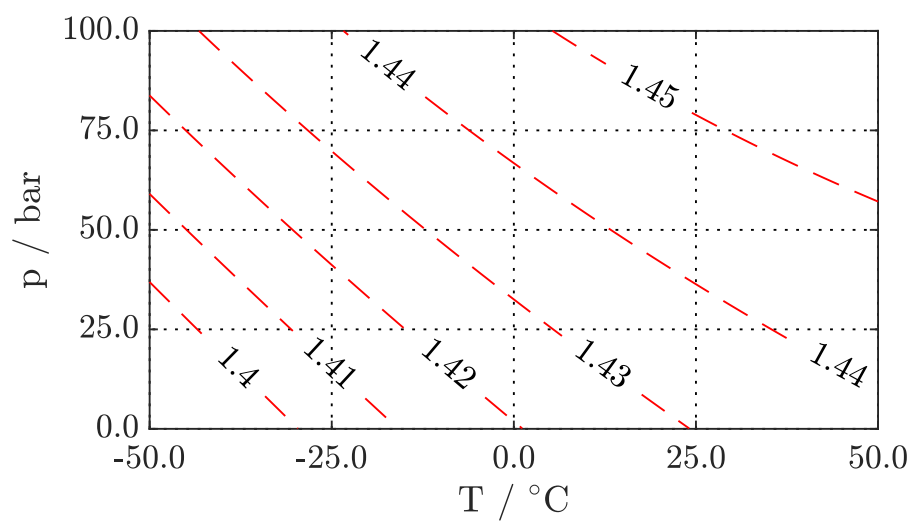

FIGURE 12: Spec. heat capacity of $\mathrm{H}_{2}$

\section{Appendix B: Analysis of pressure loss variation with $\mathrm{H}_{2}$ fraction}

Pressure loss as a function of $\mathrm{H}_{2}$ volume fraction (Fig. 7) has a rather surprising shape with a maximum at about 0.65 . That this is indeed true and foremost a result of the ratios of molar weight and heating value can be shown analytically: Pressure drop is defined as: 


$$
\Delta p=\frac{\rho}{2} u_{m}^{2} \lambda \frac{L}{d}
$$

Where the mean velocity $u_{m}$ can be expressed by the constant heating power $\dot{P}$ and the volumetric heating value $h_{u, v}$ and it follows for the pressure loss:

$$
\Delta p=\frac{\rho}{2}\left(\frac{\dot{P}}{h_{u, v}} \frac{4}{\pi d^{2}}\right)^{2} \lambda \frac{L}{d}
$$

or in terms of spec. heating value $h_{u, v}$ :

$$
\Delta p=\frac{\rho}{2}\left(\frac{\dot{P}}{h_{u} \rho} \frac{4}{\pi d^{2}}\right)^{2} \lambda \frac{L}{d}
$$

All constant values are discarded and density is expressed by the law of mixtures of ideal gases resulting in:

$$
\Delta \tilde{p}=\frac{1}{h_{u}^{2}} \frac{1}{\frac{p}{R T}} \lambda=\frac{R}{h_{u}^{2}} \lambda
$$

$\lambda$ is a complex function of Reynolds-number and hence velocity and density and expressing $\lambda$ in this analytical derivation would be a cumbersome undertaking so it is neglected for the moment. The law of mixtures of ideal gases allows to express $\Delta \tilde{p}$ as a function of $\mathrm{H}_{2}$ spec. mass fraction:

$$
\Delta \tilde{p}=\frac{\zeta R_{H_{2}}+(1-\zeta) R_{C H_{4}}}{\left(\zeta h_{u, H_{2}}+(1-\zeta) h_{u, C H_{4}}\right)^{2}} \lambda
$$

From the first derivative of this equation with respect to the mass fraction $\zeta$ the maximum pressure drop is given by:

$$
\zeta=\frac{h_{u, C H_{4}}}{h_{u, H_{2}}-h_{u, C H_{4}}}-2 \frac{R_{C H_{4}}}{R_{H_{2}}-R_{C H_{4}}}
$$

It follows that a maximum pressure loss occurs at a mass fraction of 0.26 respectively volume fraction of 0.74 and that the pressure loss for pure $\mathrm{CH}_{4}$ and pure $\mathrm{H}_{2}$ are similar. This is the same trend as shown in Fig. 7 where, in addition, $\lambda$ is a function of the inlet conditions and changes by $9 \%$. 